\title{
Interaction between treatment delivery delay and stage on the mortality from non-small cell lung cancer
}

\author{
Fernando Conrado Abrao ${ }^{1,2}$, Igor Renato Louro Bruno de Abreu ${ }^{1,2}$, Roberto Odebrecht Rocha ${ }^{1}$, Felipe \\ Dourado Munhoz ${ }^{1}$, João Henrique Godoy Rodrigues ${ }^{1}$, Bernardo Nogueira Batista ${ }^{3}$ \\ ${ }^{1}$ Department of Thoracic Surgery, Faculdade de Medicina Santa Marcelina, Rua Santa Marcelina, 155, São Paulo, Brazil; ${ }^{2}$ Department of Thoracic \\ Surgery, Oncology Center of Hospital Alemão Oswaldo Cruz, Rua Joao Juliao, 331, São Paulo, Brazil; ${ }^{3}$ Breast Unit, Hospital Sírio Libanês, Rua \\ Adma Jafet, 91, São Paulo, Brazil \\ Contributions: (I) Concept and design: FC Abrao, BN Batista; (II) Administrative support: RO Rocha, IR de Abreu; (III) Provision of study \\ materials or patient: FD Munhoz, JH Rodrigues; (IV) Collection and assembly of data: FD Munhoz, BN Batista, RO Rocha; (V) Data analysis and \\ interpretation: FC Abrao, IR de Abreu; JH Rodrigues; (VI) Manuscript writing: All authors; (VII) Final approval of manuscript: All authors. \\ Correspondence to: Fernando Conrado Abrao. Rua Sousa Ramos, 144, Apt 03, Sao Paulo (SP), Brazil. Email: fernandocabrao@uol.com.br.
}

Background: The aim of this study is to evaluate the interaction between treatment delay and stage on the mortality from non-small cell lung cancer (NSCLC).

Methods: We performed a survival analysis in a cohort of patients admitted to the reference cancer center. The following data were collected: age, gender, smoking status, tumor staging, type of lung cancer, and time from the date when the patient was diagnosed with cancer to the starting date of effective treatment. Univariable and multivariable Cox proportional hazard models were used to investigate the association between potential confounders identified during the study design. After the final adjusted model was determined, tests for interaction among all predictors were performed.

Results: Inclusion criteria were met by 359 patients. In the adjusted analysis, delayed treatment delivery was a protective factor for the risk of death, with a crude hazard ratio $(\mathrm{HR})=0.75(0.59-0.97 ; \mathrm{P}=0.02)$ and an adjusted $\mathrm{HR}=0.59(0.46-0.77 ; \mathrm{P}<0.001)$. However, a statistically significant interaction with mortality was observed between timely treatment and tumor stage. Patients with stage II disease who received delayed treatment had a higher risk of death $[\mathrm{HR}=3.08$ (1.05-9.0; $\mathrm{P}=0.04)]$. On the other hand, stage $\mathrm{IV}$ patients who received delayed treatment had a 52\% reduction in mortality $[\mathrm{HR}=0.48(0.35-0.66 ; \mathrm{P}<0.001)]$.

Conclusions: Stage of disease influenced the association between start of the treatment and mortality, and only the subgroup of stage II patients seemed to benefit from early treatment.

Keywords: Lung neoplasms; public health; mortality

Submitted Dec 08, 2017. Accepted for publication Apr 04, 2018.

doi: $10.21037 /$ jtd.2018.05.22

View this article at: http://dx.doi.org/10.21037/jtd.2018.05.22

\section{Introduction}

Lung cancer is the leading cause of cancer-related death worldwide, with 1.5 million deaths estimated in 2012 (1). These numbers are higher than the sum of deaths due to breast, prostate, and colon cancer and account for $18 \%$ of all deaths from cancers (2). Although the incidence and mortality from lung cancer have declined recently in the developed world, mostly due to reduced numbers of active smokers, countries such as Brazil are still observing a continuous increase in the incidence and lethality of lung cancer (3).

In cancer care, early detection and timely delivery of proper treatment are recognized as important strategies to improve outcomes $(4,5)$. With that purpose, different guidelines have tried to establish a limit to the timespan between diagnosis and treatment to guarantee quality in lung cancer care. The British Thoracic Society recommends 
that the time interval between diagnosis and treatment be no longer than eight weeks for surgery, seven weeks for radiotherapy, and four weeks for chemotherapy (6). The American College of Chest Physicians recommends that chemotherapy be started up to seven days after diagnosis, radiotherapy up to 4 weeks, and surgery up to 4 weeks after a surgeon's evaluation (7).

However, the current literature has failed to prove reduction in lung cancer mortality from timely treatment of cancer. In a recent study performed in the Surveillance, Epidemiology and End Results (SEER) database, including almost 40,000 lung cancer patients older than 65, authors reported a statistically significant protective effect of treatment delay, even after adjustment for stage and other potential confounders (8). This result is supported by other European studies (9-12). We performed a study to test the hypothesis that treatment before this legally-established time frame has an impact on the survival of patients with non-small-cell lung cancer (NSCLC).

\section{Methods}

We performed a survival analysis in a cohort of patients admitted to a public reference cancer center in the state of São Paulo, Brazil, between July 2008 and December 2014, with a diagnosis of lung cancer. This study was previously approved by the local Research Ethics Committee, registered under protocol 49258615.4. Potential subjects for inclusion were identified using the institution's prospectively kept Cancer Registry. Patients were considered eligible if they were 18 years or older and had not undergone any previous oncological treatment prior to admission. Histopathological reports were used to confirm lung cancer diagnosis and histological type. Patients with small-cell carcinoma, carcinoid tumors, undetermined histological type, or unresectable tumors diagnosed only after thoracotomy were excluded.

Additional clinical information was collected from the patients' medical records. These included age, gender, smoking status, tumor staging, type of treatment received (surgical versus clinical/palliative), and time from cancer diagnosis to treatment, recorded in months.

Our primary outcome of interest was death from any cause. Unfortunately, we did not have data about deaths that occurred specifically from lung cancer. Events and their dates were also determined based on our institution's Cancer Registry or by telephone contact with the patient or family. Patients who were alive at the time of their last available record were considered censored. Survival time was measured from the treatment start (day of surgery; first day of radiation, chemotherapy, or palliative care).

\section{Statistical analysis}

Descriptive analysis of the data is presented as median and interquartile range (IQR) for continuous variables and by absolute and relative frequencies for categorical variables. A survival analysis was performed to determine the effect of the measured variables on lung cancer survival after treatment. Univariable Cox proportional hazard models were used to investigate the association between potential confounders identified during the study design. If a $\mathrm{P}<0.1$ was found in the univariable analysis, the candidate predictor was included in a multivariable Cox model by either adjustment or stratification. After the final adjusted model was determined, tests for interaction between all predictors were performed. If the interaction term had a $\mathrm{P}<0.05$, a positive interaction for mortality between the predictors was considered significant. The hazard proportionality assumption of the final model was tested with Schoenfeld test. The Stata 14.2 (StataCorp, TX, USA) statistical package was used for the analysis (13), and a twotailed $\mathrm{P}<0.05$ indicated statistical significance.

\section{Results}

We identified 469 patients treated for lung cancer in the study inclusion period. Of these, 110 were excluded from the analysis (96 patients had small-cell carcinomas; 9 had carcinoid tumors; 5 patients had unresectable tumors diagnosed only after thoracotomy). Inclusion criteria were met by 359 patients, including 17 (4.7\%) patients who received optimal supportive care. Patients received treatment according to disease stage, comorbidities, and/ or performance status. Patients with stages I, II and IIIA NSCLC were treated with surgery (20.6\%). Lobectomy was the most common procedure $(n=54)$, followed by sublobar resection $(n=12)$ and pneumonectomy $(n=8)$. As mentioned above, IIIA stage $(n=18)$ cases were treated with multimodality treatment, including surgery. In IIIB stage cases $(n=55)$, the chemoradiation approach was used concomitantly or sequentially. The stage IV patients $(n=230)$ were treated with platinum-based chemotherapy. Of the 359 patients included in the study, 278 (77.4\%) died during follow-up, including one postoperative death and four deaths related to chemotherapy or radiotherapy, whereas 


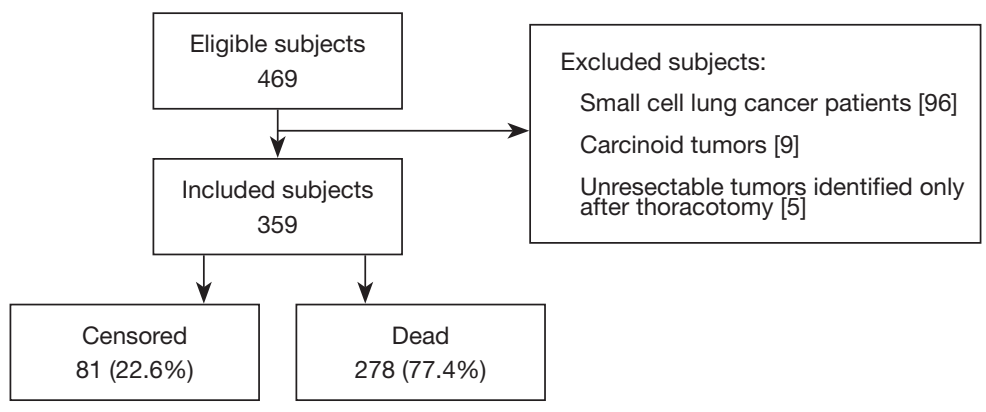

Figure 1 Flowchart describing the steps involved in creating study cohorts using the inclusion and exclusion criteria.

$81(22.6 \%)$ were alive and censored at their last recorded observation. Overall median survival time was 7 months [95\% confidence interval (CI): 6-9] (Figure 1).

Table 1 summarizes the distribution of the study variables and the hazard ratios (HR) for the survival analysis from univariable Cox models for the predictor candidates. Age, gender, and smoking status were not statistically significant predictors of mortality and were not considered for the multivariable analysis. In both the crude and adjusted analysis, delayed treatment was protective factor for the risk of death, with a crude HR $=0.75(0.59-0.97 ; \mathrm{P}=0.02)$ and an adjusted HR $=0.59(0.46-0.77 ; \mathrm{P}<0.001)$ (Table 2). Surgical treatment was associated with a $49 \%$ reduction in the risk of death after lung cancer treatment $[\mathrm{HR}=0.51(0.34-0.75)$; $\mathrm{P}=0.001]$, after adjustment.

A statistically significant interaction with mortality was observed between timely treatment and tumor stage $(\mathrm{P}=0.01)$. Table 3 and Figure 2 show the HR for mortality after delaying treatment according to the different stages, adjusted for the treatment received. For stages I and III, mortality was not significantly different between those who received treatment before or 2 months after diagnosis [stage I: HR $=1.24(0.39-3.98 ; \mathrm{P}=0.71)$; stage III: HR $=0.65(0.38-1.1 ; \mathrm{P}=0.11)]$. However, patients with stage II disease who received delayed treatment showed a 3 -fold higher mortality than those who received timely treatment $[\mathrm{HR}=3.08$ (1.05-9.0; $\mathrm{P}=0.04)]$. On the other hand, stage IV patients who received delayed treatment had a $52 \%$ reduction in mortality $[\mathrm{HR}=0.48(0.35-0.66 ; \mathrm{P}<0.001)]$.

\section{Discussion}

In this single-center cohort study including 359 patients, we detected a statistically significant interaction between treatment delay longer than 2 months after diagnosis and stage of disease regarding mortality from NSCLC. However, stage II patients benefited from timely treatment, whereas delay in treatment delivery was a protective factor for patients with stage IV disease. Moreover, surgical treatment was associated with reduction in the risk of death after lung cancer treatment.

Challenging common understandings on cancer care quality, Nadpara et al. were unable to determine an association between timely care and a better prognosis in a cohort of 37,792 elderly subjects from the SEER database (8). These authors reported a $32 \%$ decrease in NSCLC mortality risk among patients receiving delayed care, which was statistically significant even after adjustment for multiple confounders. In their stratified analysis by cancer stage, they observed better survival outcomes with timely care in patients with early-stage disease (Stage I/II), but the results were not significant. A published Canadian study analyzed 495 NSCLC patients. In a multivariable analysis, for every week of prolonged delay, the HR of survival improvement is 0.97 (95\% CI: 0.96-0.99). For each month of prolonged delay, survival improved with an HR of 0.89 (95\% CI: 0.82-0.97). For stage IV, the only significant factor associated with survival was treatment delay, as every month of treatment delay was associated with an improved survival with an HR of 0.74 (0.59-0.93), $\mathrm{P}=0.009$ (14). We found a similar crude protective effect of treatment delay, but when our analysis was stratified, we observed a significant survival improvement in stage II patients who received timely care. The protective effect of delayed treatment for stage IV patients was maintained.

On the other hand, but also surprisingly, some studies did not show any differences between timely care and survival. Nadpara $e t a l$. published another study involving NSCLC patients. The survival was not statistically different in patients receiving timely care compared to those receiving 
Table 1 Sample characteristics and univariable Cox proportional hazard models

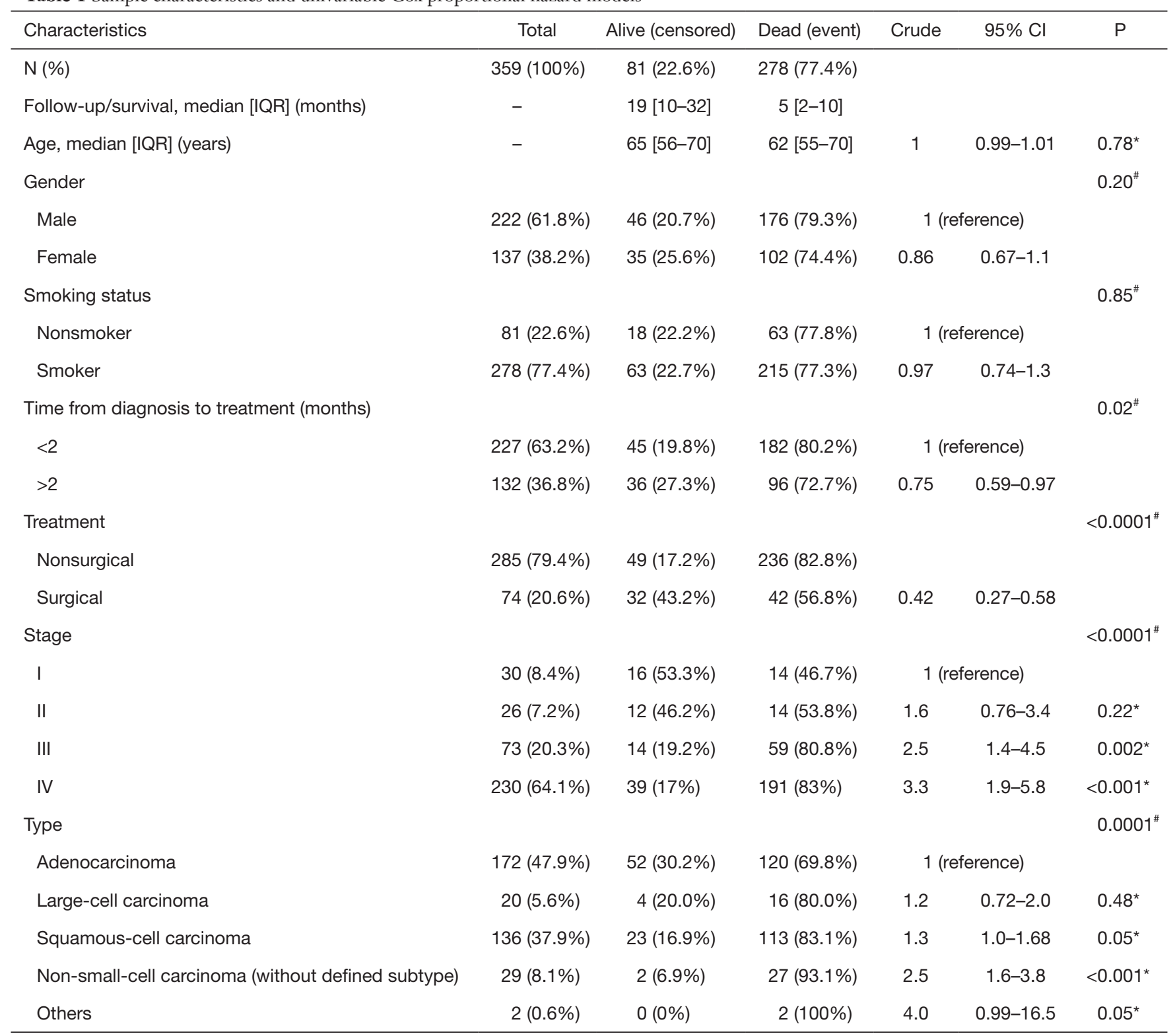

$\mathrm{Cl}$, confidence interval; IQR, interquartile range. *, Wald's Z-test; \#, log rank test.

delayed care (299 vs. 467 days). Stratified analysis by lung cancer type and stage showed similar results (15). Similarly, in Norway, a study with a national registry covering all the new cancer cases diagnosed between 1990 and 1996 included 271 patients. Factors related to survival were weight loss at the time of diagnosis, age, stage, performance status and a curative-intent surgical intervention. The authors did not find a significant association between treatment delay and survival (16).

As for stage II NSCLC patients, to the best of our knowledge, this is the first report that showed a protective association between timely care and survival. Quarterman et al. analyzed the association between survival and treatment delay between the detection and resection of early-stage lung cancers (stage I and II). The authors demonstrated no significant effect of preoperative delay on postoperative survival. The estimated HR for a 90-day increment in delay was 1.06 (95\% CI: 0.87-1.30) (17). Diaconescu et al. also evaluated the association between survival and timely care for early stage NSCLC patients. However, given the fact that 
there were relatively few events (deaths) in stage I, II and III patients, they were combined for the proportional hazards analysis. For this group with 195 patients, treatment delay was not associated with survival at the multivariable analysis (14). Another report published by Shin et al. involved 398 NSCLC patients treated through curative surgery as their first therapeutic modality. Surgical delay beyond 12 weeks, when compared to surgery performed within 1 to 4 weeks after diagnosis was not associated with increased mortality, with adjusted $\mathrm{HR}=0.79(0.42-1.48)$ (18).

A few differences in our protocols and study databases can account for the discordant findings. Timely delivery of care had different definitions in different studies. In the SEER study, treatment delay was defined as an interval

Table 2 Multivariable Cox model, adjusted by type of lung cancer; hazard of death after beginning of treatment

\begin{tabular}{|c|c|c|c|}
\hline Variables & $\mathrm{HR}$ & $95 \% \mathrm{Cl}$ & $\mathrm{P}$ \\
\hline \multicolumn{4}{|l|}{ Treatment } \\
\hline Nonsurgical & \multicolumn{2}{|c|}{1 (reference) } & - \\
\hline Surgical & 0.51 & $0.34-0.75$ & 0.001 \\
\hline \multicolumn{4}{|c|}{$\begin{array}{l}\text { Time from diagnosis } \\
\text { to treatment (months) }\end{array}$} \\
\hline$<2$ & \multicolumn{2}{|c|}{1 (reference) } & - \\
\hline$>2$ & 0.59 & $0.46-0.77$ & $<0.001$ \\
\hline Stage & & & 0.003 \\
\hline I & \multicolumn{2}{|c|}{1 (reference) } & - \\
\hline II & 1.7 & $0.76-3.7$ & 0.20 \\
\hline III & 2.2 & $1.1-4.2$ & 0.023 \\
\hline IV & 2.7 & $1.4-5.2$ & 0.003 \\
\hline
\end{tabular}

$\mathrm{Cl}$, confidence interval; HR, hazard ratio. between diagnosis and treatment lasting more than 8 weeks for surgery, 7 weeks for radiotherapy, and 6 weeks for chemotherapy. In our study, we established a cutoff of 2 months, regardless of the type of treatment offered. Although in our study only $63.2 \%$ of patients received treatment up to 2 months after the diagnosis, $77.5 \%$ of patients received timely care according to their shortertime definitions. In a Finnish study, among all patients with lung cancer, only $49 \%$ of patients received timely care according to their definitions (19). These differences in timeliness should be acknowledged when comparing results across different studies. Furthermore, the SEER database included only elderly patients ( $>65$ years.), whereas our study cohort consisted of patients aged 18 years or older. The protective effect resulting from timely delivery of these highly morbid treatment alternatives might decrease in an older population.

The protective effect resulting from treatment delay in stage IV cancer can only be explained by a tendency of health care professionals to provide faster care to patients with worse prognosis, if the choice is left to uninformed, subjective decision-making processes. Some studies have reported these results $(8,11)$. Furthermore, Diaconescu et al. compared treatment delays for the three groups of patients: localized, regional, and advanced. The advanced group had the shortest treatment delay of 50 days when compared with the localized and regional groups, who had a median treatment delay of around 3 months $(\mathrm{P}=0.001)(14)$. This risk of bias is very important because almost two thirds of the patients in our cohort were diagnosed at this more advanced stage. On the other hand, as mentioned above, these authors reported the association between survival and timely care with a more homogeneous sample regarding stage and found similar results. The study reported on 195 patients with local-regional (39.8\% at stage I-II and 21.2\% at stage III) and $124(39 \%)$ at stage IV. For patients with

Table 3 Interaction between treatment delay and stage in the Table 2 model; hazard ratio for mortality according to timely treatment, across different stages of lung cancer

\begin{tabular}{|c|c|c|c|c|}
\hline Delay & \multicolumn{4}{|c|}{ HR (95\% Cl); P } \\
\hline$<2$ months & 1 (reference) & $1.14(0.38-3.47) ; P=0.82$ & 2.73 (1.16-6.40); $P=0.02$ & 3.77 (1.65-8.63); $P=0.002$ \\
\hline$>2$ months & 1.24 (0.39-3.98); $P=0.71$ & 3.52 (1.24-9.95); $P=0.02$ & 1.76 (0.72-4.30); $P=0.21$ & $1.80(0.76-4.26) ; P=0.18$ \\
\hline $\begin{array}{l}\text { Within stage effect of delay in } \\
\text { treatment }\end{array}$ & $1.24(0.39-3.98) ; P=0.71$ & 3.08 (1.05-9.0); P=0.04 & 0.65 (0.38-1.1); P=0.11 & $0.48(0.35-0.66) ; P<0.001$ \\
\hline
\end{tabular}

$\mathrm{Cl}$, confidence interval; HR, hazard ratio. 

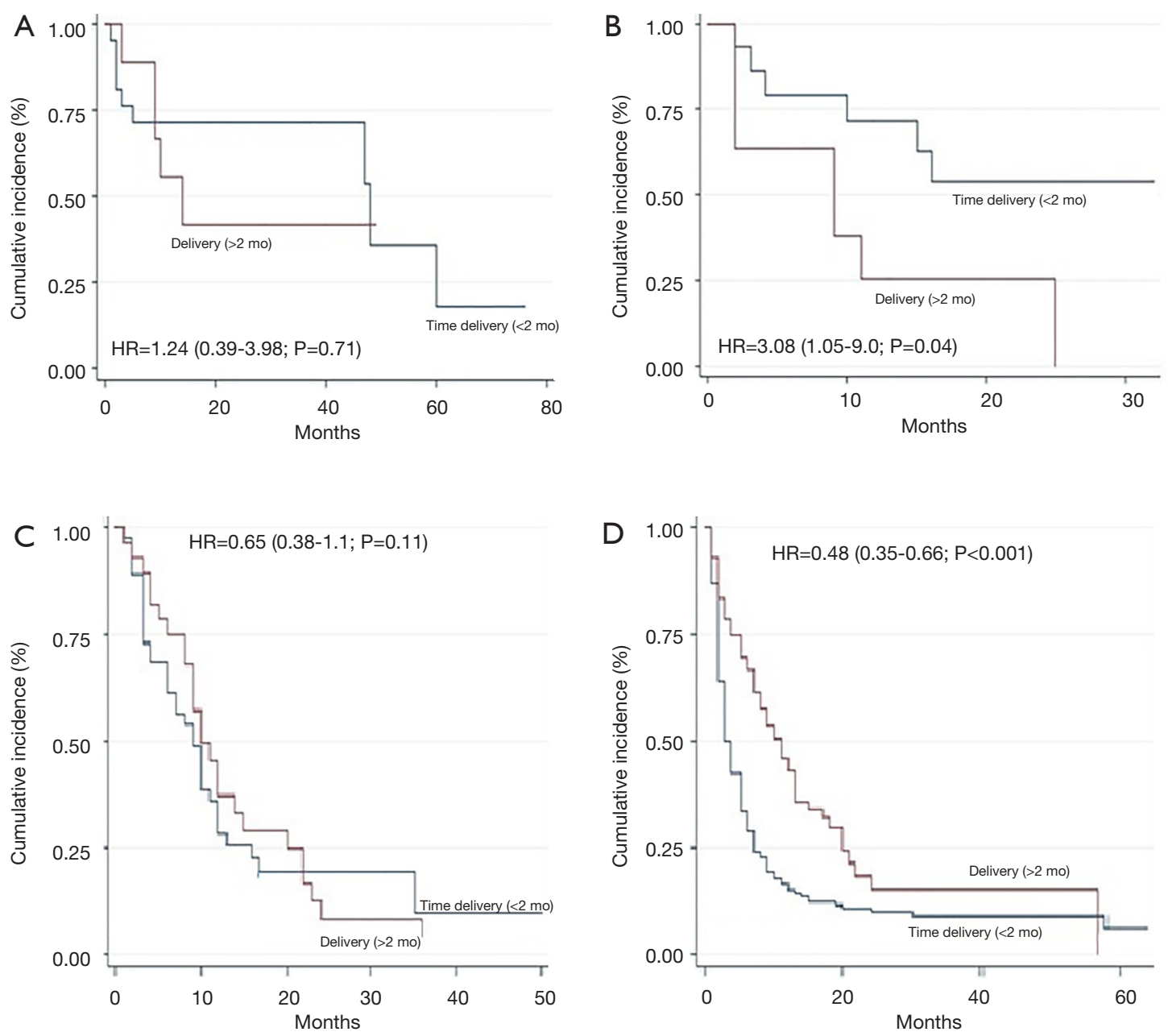

Figure 2 Kaplan-Meier curves of overall survival in patients with NSCLC according to treatment delay and stratified by stage. (A) Overall survival in all patients with stage I NSCLC; (B) overall survival in all patients with stage II NSCLC; (C) overall survival in all patients with stage III NSCLC; (D) overall survival in all patients with stage IV NSCLC. HR and 95\% CI are for timely treatment delivery ( $<2$ months) versus delayed treatment ( $>2$ months). P values comparing risk groups were calculated using the log-rank test. NSCLC, non-small cell lung cancer.

loco-regional disease, the only significant factor associated with survival was age. However, for stage IV patients, treatment delay was associated with better survival, with HR for survival being $0.93(0.88-0.98)$ (14). As for stage I and III, we were unable to determine significant improvement in survival after timely delivery of treatment. Our study is probably underpowered to detect smaller survival benefits for these subjects. Alternatively, we could hypothesize that the proposed time frame of 2 months does not have the same significance for a stage II patient, who will progress to advanced disease faster.

\section{Conclusions}

Our findings suggest, for the first time, that providing timely care can have a positive impact on the survival of patients with localized disease (stage II). Additionally, our report suggests that the time interval between diagnosis and treatment determined by the British Thoracic Society as a goal in lung cancer care is significant as an indicator of lung cancer care quality, especially for patients with localized disease. If confirmed in a larger series, our findings can provide important information that can be used to plan 
the delivery of lung cancer care, especially in high-volume hospitals for lung cancer patients.

\section{Acknowledgements}

None.

\section{Footnote}

Conflicts of Interest: The authors have no conflicts of interest to declare.

Ethical Statement: This study was previously approved by the local Research Ethics Committee, registered under protocol 49258615.4 and Informed Consent was not required.

\section{References}

1. American Cancer Society. Global Cancer Facts \& Figures 3rd edition. Atlanta: American Cancer Society, 2015.

2. Jemal A, Bray F, Center MM, et al. Global cancer statistics. CA Cancer J Clin 2011;61:69-90. Erratum in: CA Cancer J Clin 2011;61:134.

3. Araujo LH, Baldotto CS, Zukin M, et al. Survival and prognostic factors in patients with non-small cell lung cancer treated in private health care. Rev Bras Epidemiol 2014;17:1001-14.

4. Asch SM, Kerr EA, Hamilton EG, et al. Quality of care for oncologic condition and HIV: a review of the literature and quality indicators. Available online: http://www.dtic. mil/dtic/tr/fulltext/u2/a386071.pdf

5. National Lung Screening Trial Research Team, Aberle DR, Adams AM, et al. Reduced lung-cancer mortality with low-dose computed tomographic screening. N Engl J Med 2011;365:395-409.

6. The Lung Cancer Working Party of the British Thoracic Society Standards of Care Committee. BTS recommendations to respiratory physicians for organising the care of patients with lung cancer. Thorax 1998;53: S1-S8.

7. Alberts WM, Bepler G, Hazelton T, et al. Lung cancer. Practice organization. Chest 2003;123:332S-7S.

8. Nadpara P, Madhavan SS, Tworek C. Guidelineconcordant timely lung cancer care and prognosis among elderly patients in the United States: A population-based study. Cancer Epidemiol 2015;39:1136-44.
9. Annakkaya AN, Arbak P, Balbay O, et al. Effect of symptom-to-treatment interval on prognosis in lung cancer. Tumori 2007;93:61-7.

10. Comber H, Cronin DP, Deady S, et al. Delays in treatment in the cancer services: impact on cancer stage and survival. Ir Med J 2005;98:238-9.

11. Myrdal G, Lambe M, Hillerdal G, et al. Effect of delays on prognosis in patients with non-small cell lung cancer. Thorax 2004;59:45-9.

12. Neal RD, Allgar VL, Ali N, et al. Stage, survival and delays in lung, colorectal, prostate and ovarian cancer: comparison between diagnostic routes. Br J Gen Pract 2007;57:212-9.

13. Stata Statistical Software: Release 14. College Station, TX: StataCorp LP, 2015.

14. Diaconescu R, Lafond C, Whittom R. Treatment delays in non-small cell lung cancer and their prognostic implications. J Thorac Oncol 2011;6:1254-9.

15. Nadpara PA, Madhavan SS, Tworek C. Disparities in Lung Cancer Care and Outcomes among Elderly in a Medically Underserved State Population-A Cancer Registry-Linked Database Study. Popul Health Manag 2016;19:109-19.

16. Skaug K, Eide GE, Gulsvik A. Predictors of longterm survival of lung cancer patients in a Norwegian community. Clin Respir J 2011;5:50-8.

17. Quarterman RL, McMillan A, Ratcliffe MB, et al. Effect of preoperative delay on prognosis for patients with early stage non-small cell lung cancer. J Thorac Cardiovasc Surg 2003;125:108-13; discussion 113-4.

18. Shin DW, Cho J, Kim SY, et al. Delay to curative surgery greater than 12 weeks is associated with increased mortality in patients with colorectal and breast cancer but not lung or thyroid cancer. Ann Surg Oncol 2013;20:2468-76.

19. Salomaa ER, Sällinen S, Hiekkanen H, et al. Delays in the diagnosis and treatment of lung cancer. Chest $2005 ; 128: 2282-8$.

Cite this article as: Abrao FC, de Abreu IR, Rocha RO, Munhoz FD, Rodrigues JH, Batista BN. Interaction between treatment delivery delay and stage on the mortality from nonsmall cell lung cancer. J Thorac Dis 2018;10(5):2813-2819. doi: 10.21037/jtd.2018.05.22 